\title{
The role of memory in the construction of linear orderings
}

\author{
PAUL W. FOOS \\ Florida International University, North Miami, Florida 33181 \\ and \\ MARK A. SABOL \\ Bowling Green State University, Bowling Green, Ohio 43402
}

\begin{abstract}
Two experiments examined the construction of linear orderings (e.g., ABCD) from presented pairs of adjacent items (e.g., AB, BC, CD) in an attempt to determine why some orders of presentation are more difficult than others. Experiment 1 found order effects when participants constructed orderings but not when they simply recalled presented pairs, suggesting that order effects reflect processing difficulties encountered during construction. Experiment 2 used several different orders and successive and simultaneous visual presentation of pairs. Results showed that order effects can be attributed to the memory load imposed by certain pairs, the type of processing demanded, the need to reorder previously stored information, and the loss of information with continued rehearsal. Results strongly support the Foos, Smith, Sabol, and Mynatt (1976) model of linear construction.
\end{abstract}

Several recent studies have presented individuals with information about adjacent items in a linear ordering (e.g, $\mathrm{AB}, \mathrm{BC}, \mathrm{CD})$ and asked the individuals to construct the ordering (e.g., ABCD). Performance is affected by the order in which information is presented (Foos, Smith, Sabol, \& Mynatt, 1976), the strategy that participants adopt in representing presented information (Mynatt \& Smith, 1977), the completeness of the underlying ordering (Foos, 1980), the number of items in the ordering (Foos, 1980), the relationship between a sentence adjective and an item's placement (Potts \& Scholz, 1975), and various combinations of the above (Foos, 1980). The present paper examines the effects produced by presentation order in an attempt to determine whether these effects can be attributed to the need for different processing for different inputs or simply to differences in the amount of information that must be held in store pending recall.

Foos et al. (1976) describe three major effects produced by the order in which adjacent pairs of items are presented to persons attempting to construct the underlying ordering. The first of these occurs when pairs of items, after the initial pair, contain one old (i.e., previously presented) and one new item. These are called match $(M)$ pairs, since they allow the individual

The authors wish to thank an anonymous reviewer for helpful suggestions in preparation of the final version of this article and to acknowledge the support of National Science Foundation Grant BNS 77-16860 awarded to Bowling Green State University, Kirk H. Smith and Barbee T. Mynatt, principal investigators. Requests for reprints should be sent to PaulW. Foos, Department of Psychology, Florida International University, Bay Vista Campus, North Miami, Florida 33181 to match the input pair with previously stored information via the old item. If an individual has stored some information (e.g., BC), there are two kinds of $M$ pairs that can be presented. In one case, the input will add a new item to the end of the previous information string (e.g., $C D$ to produce $B C D$ ), and in the other case, to the beginning (e.g., $\mathrm{AB}$ to produce $\mathrm{ABC}$ ). The former is called Process M1, and the latter, Process M2. Presentation orders produce better performance as the number of $M 1$ pairs exceeds the number of $M 2$ pairs.

The second effect of presentation order occurs when all the pairs are not $M$ pairs. When one presents a pair of new, and thus nonmatching, items, performance declines. After some initial information is stored (e.g., $A B)$, an $M$ pair adds one new item to memory, whereas a nonmatch pair (e.g., CD) adds two. It has been suggested (Foos et al., 1976; Kieras, 1978) that the difficulty with orders containing a nonmatch pair is in part due to this increased memory load.

The final effect of presentation order is a difference between two types of double match (D) pairs. A D pair consists of two old items and allows integration of an initial pair and later nonmatch pair into a single string of items. The order of the two items in the $\mathrm{D}$ pair may be the same as the order in which they occurred in initial and nonmatch pairs or it may be reversed. The former is said to be a confirming double match and the latter is referred to as a disconfirming double match. For example, if the individual has stored $\mathrm{AB}$ followed by $\mathrm{CD}$, the double match $\mathrm{BC}$ is confirming and its processing is labeled $\mathrm{D} 1$. If the individual has stored $\mathrm{CD}$ followed by $\mathrm{AB}$, the $\mathrm{BC}$ pair is disconfirming and its processing is labeled D2. Generally, orders containing D1 pairs 
produce better performance than those with D2 pairs, although the representational strategy adopted by individuals makes a difference (Foos, 1980; Mynatt \& Smith, 1977).

The Foos et al. (1976) model of linear construction assumes that these order effects occur because the presented pairs demand different kinds of processing (e.g., M1 vs. M2; D1 vs. D2) or produce different storage requirements (e.g., match vs. nonmatch). There is, however, another explanation.

Presentation order effects could be due to lower recall of some sequences of repeated items. If individuals constructing linear orderings do not begin construction until the last pair has been presented, they may be assumed to hold a sequence of disconnected items, some of which are repeated. For example, an order containing only M1 pairs would leave the individual with a sequence of items like $A B B C C D$, whereas a nonmatch order with a D2 pair would leave a sequence like CDABBC. Wicklegren (1965) has shown that different sequences of repeated items produce different levels of recall. Thus, certain presentation orders may make string construc. tion more difficult because they lead to different input sequences of repeated items, which in turn leads to different levels of recall for those repeated items. When repeated items are recalled well, string construction is easy. When repeated items are forgotten, string construction is difficult.

To test this hypothesis, Experiment 1 used the presentation orders shown in Table 1. These include two match orders, one with all M1 pairs and one with all M2 pairs, and four nonmatch orders, two containing D1 pairs and two containing D2 pairs. Besides constructing underlying orderings, participants were given a session in which they were told to simply recall the presented pairs of items. The Foos et al. (1976) model predicts the three order effects for the construction but not for the recall task. That is, M1-M2 and D1-D2 processing differences only occur when processing takes place. The memory load of a nonmatch order is only greater than a match order when the latter is integrated to form a single string of items. With no construction, the task of recalling pairs of presented items should be unaffected by the order of presentation unless order effects are due to differences in recall produced by different input sequences.

\section{EXPERIMENT 1}

\footnotetext{
Method

Subjects. Forty introductory psychology students fulfilling a course requirement served as subjects. Each subject received all experimental conditions.

Materials. The orderings to be constructed by subjects were composed of 5 digits randomly chosen from a 10-digit pool Presentation orders are shown in Table 1. Half of the subjects received a session of string construction followed by a session in which they were told to simply recall the presented pairs. The other half of the subjects received the pair recall sessions first, followed by a string construction session. In each of these
}

sessions, each of the six presentation orders occurred three times for a total of 18 trials/session and 36 trials overall.

Procedure. All participants were run in small groups of two to six persons. Participants were told to listen to the presented pairs of digits for later recall or to try to construct the underlying linear ordering. In the latter case, the experimenter made it clear that the first-heard member of a pair preceded the second-heard member in the underlying ordering by using the presentation order $\mathrm{BC}, \mathrm{CD}, \mathrm{AB}$ as an example. All pairs were read with normal intonation at a 3-sec rate. After presentation of the final pair on a trial, the experimenter waited $3 \mathrm{sec}$ before giving a recall signal. Participants were allowed $15 \mathrm{sec}$ to write down the constructed string or remembered pairs on data sheets provided by the experimenter. After $15 \mathrm{sec}$, the experimenter gave a verbal ready signal and waited $3 \mathrm{sec}$ before presenting the first pair of the next trial.

\section{Results and Discussion}

Table 1 shows the proportion of correctly constructed orderings. For the recall task, a correct response means all presented pairs were recalled. The overall effect of session order (i.e., pair recall first vs. string construction first) was not significant $(\mathrm{F}<1)$ and did not interact with type of task $[F(1,38)=1.89]$, presentation order $(\mathrm{F}<1)$, or Task by Order factors $(\mathrm{F}<1)$. Remaining analyses were a set of planned orthogonal comparisons performed on the data collapsed over order of sessions. All comparisons were conducted at the 5\% level of significance. The overall effect of type of task was not significant $[F(1,39)=1.84]$.

The critical tests of constructive (Foos et al., 1976) and nonconstructive explanations of presentation order effects are to be found in the Task by Order interactions. Since Foos et al. (1976) attribute these effects to different constructive processes, the effects should be present during construction but not during simple recall. Thus, match-nonmatch, M1-M2, and D1-D2 comparisons should interact with type of task. On the other hand, if order effects are produced by different levels of recall due to different sequences of repeated items, then none of the above interactions should be significant. Since

Table 1

Mean Proportion of Correct Constructions and Recall, Experiment 1

\begin{tabular}{cccc}
\hline & \multicolumn{3}{c}{ Task } \\
\cline { 2 - 4 } Order & $\begin{array}{c}\text { String } \\
\text { Construction }\end{array}$ & $\begin{array}{c}\text { Pair } \\
\text { Recall }\end{array}$ \\
\hline & \multicolumn{3}{c}{ Match } \\
1. AB, BC, CD, DE (3) & .81 & & .52 \\
2. DE, CD, BC, AB (0) & .33 & & .39 \\
& & D1 & \\
3. AB, CD, DE, BC (1) & .48 & & .41 \\
4. AB, DE, CD, BC (0) & .33 & & .38 \\
& & D2 & \\
5. CD, DE, AB, BC (1) & .18 & & .55 \\
6. DE, CD, AB, BC (0) & .23 & & .44 \\
\hline
\end{tabular}

Note-For purposes of illustration, the correct ordering is always $A B C D E$. The number in parentheses is the total number of $M I$ pairs for that order. 
the sequences are the same in both tasks, the order effects should also be the same.

Results strongly support the Foos et al. (1976) model. Task produced a significant interaction with match-nonmatch differences $[F(1,39)=28.27, p<.01]$, M1-M2 differences $[F(1,39)=17.21, p<.01]$, and D1-D2 differences $[F(1,39)=19.43, p<.01]$. Mean proportions of correctly constructed orderings for match and nonmatch orders were .57 and .30 , respectively. Mean proportions of correctly recalled pairs for match and nonmatch orders were .46 and .44 , respectively. Since not all comparisons of interest were tested with the planned comparisons, the NewmanKeuls test $(\alpha=.05)$ was used on the set of 12 (i.e., 6 orders $\times 2$ tasks) treatment means. This test revealed no significant differences among the six presentation orders on the pair recall task. The expected differences between M1-M2 and D1-D2 orders were significant on the construction task.

It seems clear that presentation order effects are not due to differences in memory for repeated items caused by variations in the input sequence (Wickelgren, 1965). The obtained order effects are those predicted by the Foos et al. (1976) model and occur only when participants are actively engaged in constructing the underlying ordering.

\section{EXPERIMENT 2}

Since order effects are clearly due to processing during the construction of an underlying ordering, attention can now be turned to that processing. The present experiment examines some of these processing difficulties by altering the presentation order and memory load imposed on the participant. A reduction in memory load is achieved by using visual stimulus presentation procedures derived from studies employing conventional concept-formation tasks (Cahill \& Hovland, 1960 ). The first type of presentation is referred to as successive presentation. In this condition, each pair of items is presented visually and then removed when the next pair is presented. In terms of memory load, this condition is analogous to the auditory presentation procedures of previous studies (the present Experiment 1; Foos et al., 1976). The second type of presentation is referred to as simultaneous presentation. This condition should greatly reduce memory load, since each pair, once presented, remains visible until the last pair for that trial has been presented. Thus a subject can continually scan the display of presented materials while constructing the inderlying ordering. These procedures and the 12 presentation orders shown in Table 2 are designed to answer several questions concerning the constructive processes outlined earlier.

The first question centers on the difficulty produced by a nonmatch pair. Previous work (Foos et al., 1976; Kieras, 1978) has suggested that a major difficulty with nonmatch pairs is the increase in memory load that they produce. Each nonmatch pair adds two new items to memory, whereas an $\mathrm{M}$ pair adds one. In addition, a nonmatch pair must be held apart from other pairs, whereas an $M$ pair allows construction of a single ordering. If this increase in memory load is a major source of nonmatch difficulty, then the reduction in load occurring in the simultaneous condition should reduce the size of any match-nonmatch difference. The difference between M1 (Order 1) and M2 (Order 2) pairs should be unaffected by the presentation procedure, since this difference is assumed to be due to the type of constructive process each pair demands rather than differences in memory load.

A second set of predictions centers on the double match processes discussed earlier. Before presentation of a $\mathrm{D}$ pair, it has been assumed that the individual holds an initial representation and a nonmatch pair in memory, according to the chronological order of their receipt (Foos et al., 1976). A marker indicating that the pairs cannot yet be united is retained between the two sets of information. (In the following, this marker is represented by a hyphen.) When a double match is presented, the individual searches the stored representation and finds two matching items. With this new information, the individual is able to drop the marker and construct a single ordering. The success of that construction depends upon the type of D pair that has been presented. For the typical rehearsing participant (Mynatt \& Smith, 1977), a D2 pair produces more difficulty than a D1 pair. The presentation conditions described above and Orders 3-12 in Table 2 are designed to locate the source(s) of this D2 difficulty. The difficulty produced by a D2 pair may reside in the search for matching items, the level of prior memory load, rehearsal-generated interference, or simple item loss during construction.

Search for matching items. One possibility is that the search for matching items is more difficult with a D2 than with a D1 pair. Using the method introduced by Sternberg (1966), Baumgarte and DeRosa (1973) had individuals memorize sets of items and then respond to two-item probes. Participants took longer to respond and made more errors when the items were in different orders in probe and set and when the set items matching the probe were separated by other items. The same situation seems to occur with a D2 pair. For example, after storing initial and nonmatch pairs, such as GB-XL, the items in a D2 pair, like LG, are in a different order, and the previously stored, matching items are separated by other items and a marker. With a D1 pair (e.g., BX), items are in the same order as previously presented and matching items are separated by a marker only. If search difficulty is a source of D1-D2 differences, then those differences may be reduced in the present simultaneous condition, in which all items remain available for visual inspection. A test of this hypothesis can be found in a comparison of Order 11, containing D1 pairs, and Order 12, containing D2 pairs.

A major difference between construction following a D2 pair compared with that following a D1 pair is in the need to reorder previously stored information 
following a D2 pair. If the individual has stored GB-XL and receives the D1 pair BX, the marker can be removed to produce the single, correct ordering GBXL. With the D2 pair LG, the marker can be removed, but the previous information must also be reordered to produce the single, correct ordering XLGB. Orders 3-10 are designed to answer questions concerning this reordering difficulty.

Prior memory load. Orders 3, 4, and 5 each contain a nonmatch pair followed immediately by a D2 pair. The three orders differ in that these two pairs occur progressively later in the presentation. These orders thus require processing of the D2 pair under three different conditions of prior memory load correspond. ing to the length of the string held in memory when the nonmatch pair is presented. If D2 processing is difficult because it requires space in memory in which to manipulate the positions of items, performance on Orders 3-5 should be progressively worse.

Rehearsal-generated interference. Orders 5-7 and 8-10 test the hypothesis that a difficulty with D2 pairs is due to interference arising from rehearsal of the incomplete ordering. If a nonmatch pair is placed in memory following an initial pair(s), rehearsal of this discontinuous sequence may result in loss of information (i.e., the marker) concerning the point of discontinuity. For example, after storing initial (e.g., GB) and nonmatch (e.g., XL) pairs, marker loss would leave the individual with a single string of items (e.g., GBXL). With a D1 pair (e.g., BX), this should cause no difficulty, but with a D2 pair (e.g., LG), one may now be unable to decide which reordering (e.g., LGBX, XLGB, BXLG) is correct. Such loss should be greatest when the item preceding the nonmatch pair remains the same with each rehearsal, producing an association between the preceding item (e.g., B) and the first item of the nonmatch pair (e.g., X). This is the case in Orders 8-10. The orders differ in how many times (i.e., one, two, or three) the erroneous link is rehearsed before the D2 pair is presented. If one cause of the difficulty with D2 pairs is interference caused by erroneous association and corresponding marker loss, performance on Orders 8.10 should be progressively worse.

Simple item loss. Orders 5-7 are similar to Orders 8-10. The last pair is always a D2, and the nonmatch pair occurs progressively earlier in the presentation. Other pairs, however, add a new item immediately before the nonmatch pair with each rehearsal. The orders differ in how many different items (i.e., one, two, or three) are rehearsed as immediately preceding the nonmatch pair. Performance on these orders can thus be compared with performance on Orders 8-10. If a problem with D2 pairs is due to formation of an erroneous association, resulting in marker loss, performance on Orders 5-7 should not grow worse. If, on the other hand, the problem is due simply to loss of items in the time elapsed between nonmatch and D2 pairs, performance on Orders 5-7 should show the same decrement predicted for Orders 8-10.

\section{Method}

Subjects. Thirty-two undergraduate students fulfilling a course requirement served as subjects. Each subject received all experimental conditions.

Materials. The orderings to be constructed by subjects were composed of six consonants. The 6 consonants in each string were randomly selected from a pool of 12 consonants chosen for low auditory confusability (Conrad, 1964): J, Q, $\mathrm{T}, \mathrm{W}, \mathrm{M}, \mathrm{H}, \mathrm{R}, \mathrm{B}, \mathrm{L}, \mathrm{Y}, \mathrm{F}$, and $\mathrm{K}$. Letter pairs were presented, with the pairs separated by commas, on the Digivue graphics display screen of a Data General Corporation Nova 1220 minicomputer. In the successive condition, each presented pair remained visible only until the next pair was presented. In the simultaneous condition, each presented pair remained visible until the last pair on that trial had been presented. In each of these conditions, subjects were given 12 practice trials (i.e., 1 for each of the 12 presentation orders) followed by 48 (i.e., 12 orders $\times 4$ presentations) experimental trials. A different random selection of consonants and a different random arrangement of presentation orders were provided for each subject. Half of the subjects received the successive condition first, followed by the simultaneous condition. The other half received the two sessions in the opposite order.

Procedure. Each subject was run individually. Pairs of letters were exposed at a rate of one pair every $3 \mathrm{sec}$. Three seconds after the last pair on a trial was exposed, all pairs were erased, and the word RECALL was presented. Ten seconds after the recall sign appeared, a READY sign was presented, and $3 \mathrm{sec}$ later, the first pair of the next trial was exposed. Subjects wrote their constructed letter strings on a data sheet provided by the experimenter.

\section{Results and Discussion}

Mean proportions of correctly constructed orderings for each presentation order in each presentation condition are shown in Table 2. Participants' responses were scored by pairs and by entire six-letter ordering. In scoring by pairs, the order of presentation, for example, $\mathrm{AB}, \mathrm{BC}, \mathrm{CD}, \mathrm{DE}, \mathrm{EF}$, was compared with the participant's response, for example, BCDEAF. Moving from left to right, each pair in the presentation order was scored one if the two letters appeared together, in the correct order, anywhere in the response. If the two letters did not appear in the response, were not adjacent, or were in the reverse order that pair was scored zero. The example given above would thus be scored 01110 . Scoring by the entire six-letter ordering (i.e., the Table 2 values) required for a score of one, a response in exact agreement with the correct ordering. The scores from the four trials on the same presentation order under each presentation condition were added to yield a total score ranging from zero (no trial correct) to four (correct on all trials). These total scores were the data entered into the analyses that follow.

Analysis by pairs. The analysis by pairs is designed to establish the locus of the effect of the simultaneous and successive presentation conditions. This information is provided by the data scored by pairs, since 
scoring by pairs allows description of serial position effects that can be interpreted in terms of basic memory processes. An analysis of variance over participants with serial position, presentation condition, and type of presentation order as factors was performed. Presentation orders for this analysis were divided into three types: match orders (i.e., Orders 1 and 2), single nonmatch orders (Orders 3-10), and double nonmatch orders (Orders 11-12). The three order types were weighted equally in this analysis.

Figure 1 shows the serial position curves for match and nonmatch (single and double combined) orders under both presentation conditions. Results showed significant linear $[F(1,31)=11.27, p<.01]$ and quadratic $[F(1,31)=129.41, p<.001]$ trends over serial position. Few errors were made on the initial pair, more on the last pair, and most on those in the middle. Both of these trends interacted with presentation condition $[F(1,31)=24.01, p<.001$, and $\mathrm{F}(1,31)=14.24, \mathrm{p}<.01$, respectively $]$. The successive condition yielded the classical serial position curve, showing both primacy and recency effects. The simultaneous condition showed primacy but not recency. The presence of a primacy effect under both conditions can be taken as evidence for ongoing construction (Bower, 1971). That is, the effect can be attributed to the rehearsal of a partially constructed ordering in which an initial pair is rehearsed several times as part of this ordering. Later pairs are rehearsed less often. If participants had delayed all construction until all pairs were presented, a primacy effect seems unlikely. Thus it appears that the simultaneous condition did not radically alter the participants' tendency to construct when possible (i.e., when an $\mathrm{M}$ or a $\mathrm{D}$ pair was presented).

Table 2

Mean Proportions of Correctly Constructed Orderings, Experiment 2

\begin{tabular}{|c|c|c|}
\hline \multirow[b]{2}{*}{ Order } & \multicolumn{2}{|c|}{ Presentation Condition } \\
\hline & Successive & Simultaneous \\
\hline & \multicolumn{2}{|c|}{ Match } \\
\hline \multirow[t]{2}{*}{ 2. $\mathrm{EF}, \mathrm{DE}, \mathrm{CD}, \mathrm{BC}, \mathrm{AB}(0)$} & $\begin{array}{l}.60 \\
.30\end{array}$ & $\begin{array}{l}.60 \\
.30\end{array}$ \\
\hline & \multicolumn{2}{|c|}{ Single Nonmatch } \\
\hline $\begin{array}{l}\text { 3. } \mathrm{CD}, A B, \mathrm{BC}, \mathrm{DE}, \mathrm{EF}(2) \\
\text { 4. } \mathrm{CD}, \mathrm{DE}, A B, \mathrm{BC}, \mathrm{EF}(2)\end{array}$ & $\begin{array}{l}.18 \\
.14\end{array}$ & $\begin{array}{l}.28 \\
.24\end{array}$ \\
\hline $\begin{array}{l}\text { 5. } \mathrm{CD}, \mathrm{DE}, \mathrm{EF}, A B, \mathrm{BC}(2) \\
\text { 6. } \mathrm{CD}, \mathrm{DE}, A B, \mathrm{EF}, \mathrm{BC}(2) \\
\text { 7. } \mathrm{CD}, A B, \mathrm{DE}, \mathrm{EF}, \mathrm{BC}(2)\end{array}$ & $\begin{array}{l}.19 \\
.11 \\
.13\end{array}$ & $\begin{array}{l}.23 \\
.15 \\
.17\end{array}$ \\
\hline $\begin{array}{r}\text { 8. EF, DE, CD, } A B, \mathrm{BC}(0) \\
\text { 9. EF, DE, } A B, \mathrm{CD}, \mathrm{BC}(0) \\
\text { 10. EF, } A B, \mathrm{DE}, \mathrm{CD}, \mathrm{BC}(0)\end{array}$ & $\begin{array}{l}.24 \\
.13 \\
.06\end{array}$ & $\begin{array}{l}.23 \\
.20 \\
.16\end{array}$ \\
\hline 10. $\mathrm{EF}, A B, \mathrm{DE}, \mathrm{CD}, \mathrm{BC}(0)$ & \multicolumn{2}{|c|}{ Double Nonmatch } \\
\hline $\begin{array}{l}\text { 11. } \mathrm{AB}, C D, E F, \mathrm{BC}, \mathrm{DE}(0) \\
\text { 12. } \mathrm{EF}, C D, A B, \mathrm{DE}, \mathrm{BC}(0)\end{array}$ & $\begin{array}{l}.28 \\
.03\end{array}$ & $\begin{array}{l}.46 \\
.07\end{array}$ \\
\hline
\end{tabular}

Note-For purposes of illustration, the correct ordering is always $A B C D E F$. The number in parentheses is the total number of $M I$ pairs for that order. For nonmatch orders, the nonmatch input is italicized.

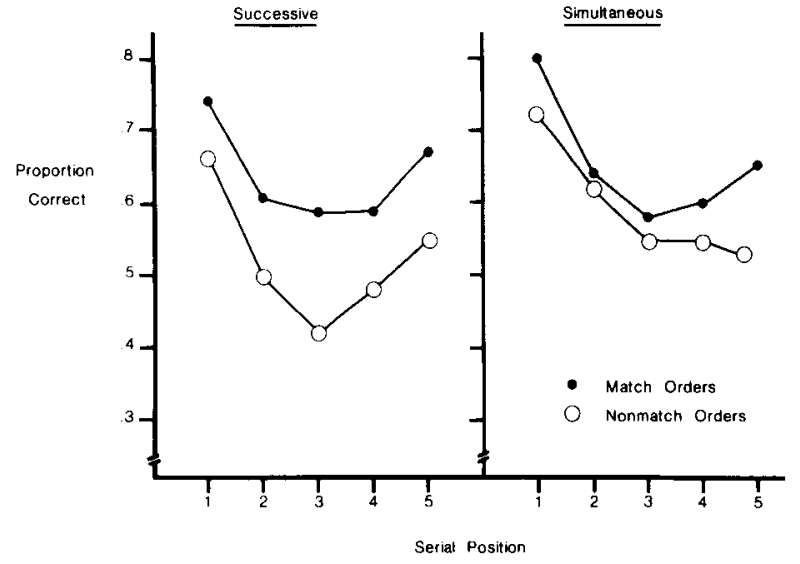

Figure 1. Serial position curves for match and nonmatch orders with successive and simultaneous presentation.

Performance on match orders was significantly better than performance on nonmatch orders $[F(1,31)=23.96$, $\mathrm{p}<.01]$. In addition, the three-way interaction among the match-nonmatch variable, successive-simultaneous presentation, and the quadratic trend over serial position was significant $[F(1,31)=8.57, p<.01]$. A close examination of Figure 1 reveals the source of this interaction. Under the successive condition, both match and nonmatch orders yielded a quadratic serial trend. Indeed, nonmatch orders showed a larger trend due to low performance on those serial positions that presented the nonmatch pair(s). Since nonmatch pairs never occur in Serial Position 1 or 5, a quadratic trend results. Under the simultaneous condition, match orders again produced a quadratic trend. Nonmatch pairs, however, no longer produced low performance and much of the quadratic trend was, therefore, absent for nonmatch orders. This improved performance on nonmatch pairs is made possible with simultaneous presentation by the reduction in memory load. From these results, it would appear that the simultaneous condition had the intended effect of reducing memory load without disrupting ongoing construction of the underlying ordering. Attention can now be turned to the data scored by entire orderings.

Analysis by orderings. The simultaneous condition produced significantly better performance than the successive condition $[F(1,30)=6.57, p<.05]$. The condition that was presented second produced significantly better performance than the condition presented first $[F(1,30)=19.41, p<.01]$, indicating a strong general practice effect. This practice did not differentially affect performance on different orders $[F(11,330)$ $=1.10]$. The interaction between presentation conditions and practice did not even approach significance $(F=0)$. This last test is critical, since a significant interaction of conditions with order of conditions would imply that only the data from each participant's first session would be usable. The zero sum of squares obtained for this test indicates that the effect of practice upon the two presentation conditions was identical. 
Remaining comparisons were performed on data collapsed over order of presentation conditions.

Performance on match orders was significantly higher than performance on nonmatch orders $[F(1,330)=$ $170.70, p<.001]$, and performance on $\mathrm{M} 1$ pairs (Order 1) was significantly higher than performance on $\mathrm{M} 2$ pairs (Order 2) $[\mathrm{F}(1,330)=58.58, \mathrm{p}<.001]$. These results replicate previous findings demonstrating the difficulty of nonmatch orders and the advantage of $M 1$ over M2 processing.

The Foos et al. (1976) model suggests that a major difficulty with nonmatch orders is the increase in memory load that occurs with presentation of a nonmatch pair. Thus, the reduction in memory load produced by simultaneous presentation should improve performance on nonmatch orders. This prediction is supported by the significant interaction between match-nonmatch orders and presentation conditions $[\mathrm{F}(1,330)=4.59$, $\mathrm{p}<.05]$. Under both conditions, match orders produced an average proportion correct of .45 . Performance on nonmatch orders increased from .15 with successive presentation to .22 with simultaneous presentation. While this increase seems fairly small, one must remember that 9 of the 10 nonmatch orders have the added difficulty produced by a D2 pair. The one order without this difficulty (Order 11) showed the greatest improvement under the simultaneous condition, resulting in performance midway between $\mathrm{M} 1$ and $\mathrm{M} 2$ match orders. It appears that when the items remain present, as they do with simultaneous presentation, the processing of a D1 pair is relatively easy, but the processing of a D2 pair is still very difficult.

The second prediction made by the model is that M1-M2 differences will be unaffected by a reduction in memory load. This prediction is clearly supported by the identical performance on Orders 1 and 2 under the two presentation conditions. Adding a new item to the beginning of a stored representation is simply more difficult than adding to the end.

Overall, performance on Order 11, containing D1 pairs, was significantly higher than performance on Order 12 , containing $D 2$ pairs $[F(1,330)=71.74$, $p<.001]$. One proposed difficulty with $D 2$ pairs is that they make the search for matching items more difficult. While the interaction between these two orders and presentation conditions was significant $[F(1,330)=5.81, p<.05]$, it clearly does not support the hypothesis of greater search difficulty. As stated above, it is Order 11, and not Order 12, that is greatly improved with simultaneous presentation. The present results should not, however, be taken as disconfirming evidence for a greater search difficulty with D2 pairs. In light of previous work (Baumgarte \& DeRosa, 1973), D2 search difficulty seems quite probable.

Orders 3-5 examine D2 difficulty under different conditions of prior memory load. That is, the nonmatch pair occurs after one (Order 3 ), two (Order 4), or three (Order 5) M pairs have been presented. If D2 difficulty is affected by the available space in memory for reordering information or the size of units to be reordered, then performance should grow progressively worse on these three presentation orders. Examination of the values in Table 2, however, reveals no such effect. There was no significant difference between Orders 3 and $4[F(1,330)=$ 1.04] . Adding Order 5 to the comparison (i.e., making it nonorthogonal with the remaining comparisons) did not alter this finding $(F<1)$.

As described previously, Orders 5-10 form two series that vary the number of pairs occurring between presentation of a nonmatch and a D2 pair. The 5-7 series presents one, two, or three different pairs that are added to the end of the initial pair. Thus, a new item is placed before the nonmatch pair after each presentation. If a problem with D2 pairs is loss of items in the time elapsed between presentations of nonmatch and D2 pairs, performance on this series should grow progressively worse. Neither linear $[F(1,330)=2.34]$ nor quadratic $[F(1,330)=2.53]$ trends, across this series, were significant. Simple item loss does not appear to account for D2 difficulty.

The 8-10 series presents one, two, or three different pairs that are added to the beginning of the initial pair. Thus, the same item is rehearsed immediately before the nonmatch pair following these one, two, or three presentations. If rehearsal leads to the formation of an erroneous association between this item and the nonmatch pair, the point of discontinuity may become blurred, and performance on this series should grow progressively worse. One clearly sees this decline in the Table 2 values, and results showed a significant linear $[\mathrm{F}(1,330)=11.36, \mathrm{p}<.01]$ but not quadratic $(\mathrm{F}<1)$ trend. An individual who has lost information concerning the point of discontinuity between initial and nonmatch pairs may be able to rediscover that point under the simultaneous condition. Thus, one might expect the linear trend to be attenuated with simultaneous presentation. Although the values in Table 2 seem to reveal such an effect, the interaction was only marginally significant $[F(1,330)=3.38, p<.10]$. None of the remaining orthogonal comparisons was significant.

\section{GENERAL DISCUSSION}

Previous work has shown that successful construction of a linear ordering is affected by the order in which information about adjacent items is presented. Foos et al. (1976) have assumed that these order effects indicate different processes operating during construction. The present experiments confirm this assumption and reveal the nature of the processing difficulties produced by different input orders.

Both experiments replicated the match-nonmatch, M1-M2, and D1-D2 differences found by Foos et al. (1976). Experiment 1, however, demonstrates that these effects occur only when participants are constructing an underlying ordering. An explanation of order 
effects in terms of poorer memory for different sequences of repeated items (Wickelgren, 1965) is thus clearly ruled out. Like the serial position effects described by Bower (1971), the above order effects indicate attempted construction of an ordering and not simply storage of presented pairs.

Experiment 2 examined the effects produced by a number of different presentation orders under successive and simultaneous presentation procedures. Results suggest that simultaneous presentation reduced the memory load imposed on participants without altering ongoing construction of the ordering. These procedures and orders provide information about the source of nonmatch, $\mathrm{M} 2$, and $\mathrm{D} 2$ difficulties.

The reduction in memory load occurring with simultaneous presentation improved performance on nonmatch but not match orders. In fact, the M1-M2 difference for match orders was identical under successive and simultaneous conditions. This result supports the hypothesis (Foos et al., 1976) that this difference results from different processing of the input. Adding to the end of a stored representation (i.e., M1) is easier than adding to the beginning (i.e., M2). For nonmatch orders, the improvement was greatest when the added difficulty of a D2 pair (i.e., Order 11) was not present. In this case, performance was the same as average match order performance. Memory load thus appears to be a major factor influencing performance on nonmatch orders.

In searching for the source of D2 difficulty, we tested several hypotheses. Changing the amount of information stored before presentation of the D2 pair did not influence D2 difficulty. It appears that the available space in memory and the size of units to be reordered are not important factors in producing lower D2 performance.

No evidence was found that would indicate a greater difficulty in finding the items matching a D2 pair than those matching a D1 pair. In light of the results presented by Baumgarte and DeRosa (1973), this lack of evidence should not be taken as disconfirmation of the search difficulty hypothesis. If greater search difficulty is a factor contributing to lower D2 performance, it probably plays a relatively minor role. As long as the other factors affecting D2 performance remain the same, easing the search for matching items would not greatly increase overall D2 performance.

A major factor producing lower D2 performance is the loss of information occurring before the D2 pair is presented. Results indicate that it is not the loss of items but the loss of information regarding the point of discontinuity between initial and nonmatch representations that is critical. Such loss occurs with continued rehearsal and leaves the individual unable to decide on a single correct ordering when the D2 pair is finally presented. The reordering operation itself adds an additional operation not present in the processing of a D1 pair.

In summary, the present results strongly support the Foos et al. (1976) model of constructive processes. Presentation order effects are clearly attributable to memory load and processing difficulties occurring with different inputs. In addition, the loss of information with continued rehearsal is a major factor producing lower performance.

\section{REFERENCES}

Baumgarte, R., \& De Rosa, D. V. Information processing in a two-item classification task: Relationships among items in a memorized set. Journal of Experimental Psychology, 1973, 100, 1.7.

Bowe r, G. H. Adaptation-level coding of stimuli and serial position effects. In M. H. Appley (Ed.), Adaptation-level theory. New York: Academic Press, 1971.

Cahill, H. E., \& Hovland, C. I. The role of memory in the acquisition of concepts. Journal of Experimental Psychology, 1960, 59, 137-144.

Conrad, R. Acoustic confusions in immediate memory. British Journal of Psychology, 1964, 55, 75-84.

Foos, P. W. Constructing cognitive maps from sentences. Journal of Experimental Psychology: Human Learning and Memory, 1980, 6, 25-38.

Foos, P. W., Smith, K. H., Sabol, M. A., \& Mynatt, B. T. Constructive processes in simple linear order problems. Journal of Experimental Psychology: Human Learning and Memory, 1976, 2, 759-766.

KiERAS, D. E. Good and bad structure in simple paragraphs: Effects on apparent theme, reading time, and recall. Journal of Verbal Learning and Verbal Behavior, 1978, 17, 13-28.

MynatT, B. T., \& Smith, K. H. Constructive processes in linear order problems revealed by sentence study times. Journal of Experimental Psychology: Human Learning and Memory, 1977, 3, 357-374.

Potts, G. R., \& Scholz, K. W. The internal representation of a three-term series problem. Journal of Verbal Learning and Verbal Behavior, 1975, 14, 439-451.

Sternberg, S. High-speed scanning in human memory. Science, 1966, 153, 652-654.

WICKELGREN, W. A. Short-term memory for repeated and nonrepeated items. Quarterly Journal of Experimental Psychology, $1965,17,14-25$.

(Accepted for publication January 30, 1981.) 